\title{
Sistematización en la producción de abono orgánico de residuos de frutas y verduras en la planta de compostaje de un gobierno municipal, Comas, 2018
}

\author{
Omar Guerrero Vargas, Eusterio Acosta Suasnábar, Elmer Benites Alfaro, María Aliaga Martínez \\ Universidad César Vallejo, Av. Av. Alfredo Mendiola 6232, Los Olivos 15314, Perú \\ Recibido 15 de junio del 2019, Revisado el 19 de julio de 2019 \\ Aceptado el 20 de julio de 2019
}

DOI: https://doi.org/10.33017/RevECIPeru2019.0006/

\begin{abstract}
Resumen
El presente trabajo de investigación propone sistematizar la producción de abono orgánico a partir de los residuos de frutas y verduras en la planta de compostaje de la Municipalidad de Comas; de tal manera mejorar la disposición final de los residuos orgánicos. Se caracterizaron los dos tipos de residuos entre frutas y verduras con pesos de $290 \mathrm{~kg}$ de hortalizas, y $72,8 \mathrm{~kg}$ de frutas, y $156 \mathrm{~kg}$ de estiércol como parte del insumo, que conforman una pila para la producción de abono. Asimismo, se utilizó otros insumos para mejoramiento de la degradación de la materia orgánica; $5 \mathrm{~kg}$ de chancaca, $100 \mathrm{~g}$ de levadura, 2,5 kg de Cal diluidas en $50 \mathrm{~L}$ de agua. Con respecto a las características fisicoquímicas medidas durante el proceso, se obtuvo como resultado la mayor temperatura de $65{ }^{\circ} \mathrm{C}$, el cual fue descendiendo hasta llegar a $29{ }^{\circ} \mathrm{C}$, el pH entre 5,8 a 7,5 durante todo el desarrollo y la humedad disminuyó acorde al proceso desde 56,57 \% hasta 36,55\%. De tal manera, con el sistema planteado para la producción de abono orgánico se obtuvo mejoras en los nutrientes esenciales del abono orgánico, con Nitrógeno $(\mathrm{N}) 1,92 \%$, Fósforo $\left(\mathrm{P}_{2} \mathrm{O}_{5}\right)$ 1,92 \% y Potasio $\left(\mathrm{K}_{2} \mathrm{O}\right) 3,19 \%$. Asimismo, pH de 7,04 y la materia orgánica (MO) $32,32 \%$ el cual estos valores mejoraron la calidad con respecto a los que se producen en la planta de compostaje de Comas.
\end{abstract}

Descriptores: Abono orgánico, sistematizar, caracterización, características fisicoquímicas, calidad.

\begin{abstract}
The present work of investigation proposes to systematize the production of organic fertilizer from the waste of fruits and vegetables in the composting plant of the Municipality of Comas; in such a way to improve the final disposal of organic waste. The two types of waste were characterized between fruits and vegetables with weights of $290 \mathrm{~kg}$ of vegetables, and $72.8 \mathrm{~kg}$ of fruit, and $156 \mathrm{~kg}$ of manure as part of the input, which make up a pile for the production of fertilizer. Likewise, other inputs were used to improve the degradation of organic matter; $5 \mathrm{~kg}$ of chancaca, $100 \mathrm{~g}$ of yeast, $2.5 \mathrm{~kg}$ of Cal diluted in $50 \mathrm{~L}$ of water. With respect to the physicochemical characteristics measured during the process, the highest temperature of $65{ }^{\circ} \mathrm{C}$ was obtained, which went down until reaching $29^{\circ} \mathrm{C}$, the $\mathrm{pH}$ between 5.8 to 7.5 during the whole development and the humidity decreased according to the process from $56.57 \%$ up to $36.55 \%$. Thus, with the system proposed for the production of organic fertilizer was obtained improvements in the essential nutrients of organic fertilizer, with Nitrogen $(\mathrm{N}) 1.92$ \%, Phosphorus (P2O5) $1.92 \%$ and Potassium (K2O) $3.19 \%$. Also, pH 7.04 and organic matter (MO.) $32.32 \%$ which these values improved the quality with respect to those produced in Comas composting plant.
\end{abstract}

Keywords: Organic fertilizer, systematize, characterization, physicochemical characteristics, quality. 


\section{Introducción}

En la actualidad vivimos en un mundo donde el crecimiento poblacional es totalmente acelerado, esto conlleva mayor generación de residuos sólidos a causa de las múltiples actividades desarrollada por el ser humano convirtiéndolo en grandes problemas ambientales.

Según (MINAN, 2016) en la implementación del Plan Nacional de Gestión de Residuos Sólidos 2016-2024; nos informa que en el 2014 Perú generó 7497482 toneladas anuales de residuos; 13244 toneladas por día y solo Lima Metropolitana y callao generan un promedio de $5870 \mathrm{TN} /$ día. Con respecto a las características de su composición, el 53,16\% son orgánicos. Por lo que se observa de acuerdo a los datos que la mayoría de los residuos generados es de material orgánico, las cuales no son reaprovechados de manera correcta, por lo que se puede darles un nuevo uso.

El distrito de Comas según el estudio de Caracterización de Residuos Sólidos Municipales, 2016 menciona, la generación de residuos es $305.130 \mathrm{TN} / \mathrm{día}$, siendo el $50 \%$ material orgánico de las cuales no se les da ningún valor a estos y terminan desechándose junto a los otros residuos para ser dispuestos en rellenos sanitarios.

La Planta de compostaje de la municipalidad de Comas está incorporando diferentes mercados de abastos para el tratamiento de sus residuos orgánicos de frutas y verduras, actualmente tiene incorporado a tres mercados, las cuales generan un promedio de 180 kg/día; 1260 kg/semana, 5040 $\mathrm{kg} / \mathrm{mes}$ que son tratados para obtener abono orgánico; Uno de las desventajas identificadas es el sistema de tratamiento, las cuales se encuentran en camas improvisadas, sin un orden adecuado, que prolongan el desarrollo del proceso desde la obtención de la materia prima hasta las obtención final, el compost.

Por ello una alternativa de solución en la planta de compostaje, es cambiar a un nuevo sistema en el proceso para poder obtener mejores resultados, tanto en tiempo y calidad del compost; de esa manera evitando que los residuos orgánicos lleguen a un relleno sanitario o simplemente sean desechados en botadero informales, los cuales empiezan a generar presencia de vectores, lixiviados, gases tóxicos nocivos para el ser humano y el medio ambiente; y una de las alternativas ya arriba mencionado es la producción de abono orgánico de manera más rápida y conservando la calidad adecuada, de esa manera devolver la fertilidad de la tierra y conservarlo para que nos siga brindado frutos.

Según Oviedo et al. [1] es su artículo "Perspectivas de aplicación del compostaje de biorresiduos provenientes de residuos municipales. Un enfoque desde lo global a o local" con el objetivo de plantear una estrategia de concientización para la aplicación del compostaje; la perspectiva que se plantean para consolidad en Colombia y los países en desarrollo, la opción de reaprovechamiento de los biorresiduos a través de procesos biológicos, para ello proponen estrategias como la separación en la fuente de recolección selectiva y de esa manera posicionar los residuos de manera adecuado para su posterior tratamiento.

Según Álvarez et al. [2] en su artículo "Rediseño y optimización de un dispositivo de compostaje a pequeña escala para ser utilizado en proyectos de agricultura urbana" con el objetivo de rediseñar y optimizar un prototipo teniendo en consideración los factores condicionantes de un proceso aeróbico de compostaje para la producción de compost de buena calidad, en esta investigación se realizó una primera fase de diagnóstico de la eficiencia del prototipo, en cuanto a la calidad del producto obtenido, e identificación de las posibles mejoras. Una segunda fase consistió en la construcción de un dispositivo rediseñado, para culminar en una última fase de experimentación y verificación de la eficiencia del mismo. Durante la realización de las distintas fases se tuvieron en cuenta siempre los factores que controlan un proceso aeróbico de compostaje (oxígeno, $\mathrm{pH}$, humedad y temperatura), así como para luego mejorar algunas características del prototipo del Jardín Botánico de Bogotá (JBB), tales como su tamaño, modo de aireación, aislamiento térmico y facilidad de volteo, entre otras, que aumentaran la eficiencia del proceso de compostaje a pequeña escala. Una vez establecidos los montajes, se procedió a la toma diaria de los parámetros de temperatura, $\mathrm{pH}$ y humedad, como punto de partida para el inicio del proceso de compostaje que tendría una duración de 28 días a partir del día de actividades de iniciación del proceso.

Según Arrigoni [4] en su tesis "Optimización del proceso de compostaje de pequeña escala" el cual tuvo como objetivo evaluar la optimización de un sistema de compostaje de pequeña escala que tenga la capacidad de adaptarse a regiones con climas fríos y permita obtener una mejora en la calidad agronómica. el cual se basó en evaluar 
cuatro estratos de altura en relación a la temperatura, las cuales fueron Tratamiento desarrollado entre 0 y $20 \mathrm{~cm}$ de altura de compostador (T10), Tratamiento desarrollado entre 20 y $40 \mathrm{~cm}$ de altura de compostador (T30), Tratamiento desarrollado entre 40 y $60 \mathrm{~cm}$ de altura de copostador (T50) y Tratamiento desarrollado entre 60 y $80 \mathrm{~cm}$ de altura de compostador (T70), sugiere que se ocasionan un efecto negativo por la estratificación y la mitigación de lixiviados hacia las partes inferiores causando la compactación; el cual quita el proceso de eficiencia; pero no descarta que la mezcla inferir compactada es mejor ya que se da mejor el proceso de saneamiento, degradación y transformación de la materia orgánica, por lo tanto la formación de los estratos cuanto más altura tengan tendrá una mejor eficiencia en el desarrollo del proceso. Ya que también de esta manera se incrementa la temperatura que es esencial para su desarrollo.

Según Larreategui et al. [5] en su revista titulado "un modelo matemático para la reducción del tiempo de compostaje" sintetiza lo siguiente; mediante una técnica de digestión de materia orgánica el cual el tiempo que demora tradicionalmente es de 16 a 24 semanas, ya que es no llega ser una manera eficiente de aprovecha los residuos orgánicos, es por ello mediante la optimización del proceso de compostaje se busca reducir en el tiempo de obtención y de buena calidad, las frutas y vegetales fueron las variables que se estudiaron al igual que un tipo de bioacelerador (levadura y microorganismos autóctonos). Para el desarrollo del proceso se hizo mediante el Diseño Factorial Completamente Aleatorio (DFCA) llegándose obtener compost de gran calidad en un tiempo de 7 semanas. Las mejores condiciones que permitieron el correcto desarrollo de operación fueron la temperatura, densidad, humedad, $\mathrm{pH}$, relación de carbono-nitrógeno dentro de la comunidad San Gabriel del Baba (Santo Domingo de los Colorados, Ecuador) de tal manera como resultado final se determinó un modelo matemático el cual detalla la relación de la temperatura y el tiempo de digestión de la materia orgánica. También se destaca la formulación del compost obtenida con residuos vegetales y microorganismos aislados de un bosque muy húmedo premontano. En este producto se encontraron concentraciones relevantes de Carbono $(10,13 \%)$ y Nitrógeno $(0,96 \%)$, fosforo $(0,86)$, Potasio $(1,63)$. En contenido de materia orgánica, se registró un porcentaje final de 27,48 \%. Este resultado remarca la importancia de incluir residuos vegetales en el compostaje por su contenido celulósico. El análisis estadístico comprueba la incidencia que origina el tratamiento con Solución Activa por Microorganismos Efectivos (EMAS) y residuos vegetales en función de la temperatura óptica de compostaje. Este modelo matemático es de importancia para el cálculo de consumo de materia prima y predicción de las condiciones de operación. p.36.

Objetivo General, fue sistematizar la producción de abono orgánico a partir de los residuos de frutas y verduras en la Planta de compostaje de la Municipalidad de Comas, 2018. Los Objetivos específicos fueron: Conocer los componentes de residuos orgánicos presentes en la planta de compostaje de la Municipalidad de Comas, 2018. Utilizar los insumos intervinientes para el sistema planteado en la producción de abono orgánico a partir de los residuos de frutas y verduras en la planta de tratamiento de la municipalidad de Comas, 2018. Medir las características fisicoquímicas para el sistema planteado en la producción de abono orgánico a partir de residuos de frutas y verduras en la planta de compostaje de la municipalidad de Comas, 2018. Evaluar la calidad del abono orgánico producido por el sistema planteado a partir de los residuos de frutas y verduras en la planta de compostaje de la municipalidad de Comas, 2018.

\section{Metodología}

\subsection{Diseño de investigación}

Diseño de tipo experimental y aplicativa de enfoque cuantitativo ya que se manipulará la variable independiente para el desarrollo del proceso. La ventaja de este tipo de estudio es poder alcanzar las condiciones adecuadas que busca esta investigación.

\subsubsection{Sistema aplicado}

Este método en un sistema nuevo el cual se basa en los siguientes estudios, como un modelo a seguir, mejorando el proceso para obtener un producto en menos tiempo y de buena calidad.

De acuerdo a la Organización de las Naciones Unidas para la Alimentación y la Agricultura (FAO), 2011 nos da las especificaciones técnicas para poder desarrollar de manera correcta el proceso en la producción del abono orgánico. El cual se tomará en cuenta para la aplicación en el nuevo sistema planteado, que mejorará de manera significativa y poder obtener un producto de calidad. 
2.1.2 Diseño de elaboración de pila de compostaje del nuevo Sistema

El diseño del armado de pila consiste en aplicar capas entre los residuos vegetales, estiércol y poda, de esta manera colocando uno encima del otro hasta obtener una altura adecuada donde pueda favorecer al aumento y conservación de la temperatura.

\section{Resultados}

Según los objetivos propuestos se plantean los siguientes resultados:

1. Caracterizar los componentes orgánicos presentes en los residuos de frutas y verduras en la planta de compostaje de la Municipalidad de Comas, 2018.

La tabla 1, indica los valores de pesos de residuos orgánicos presentes que se obtuvo para la elaboración del compost entre frutas y verduras, provenientes de los mercados Sangarará, La Alborada y Mi Hacienda.

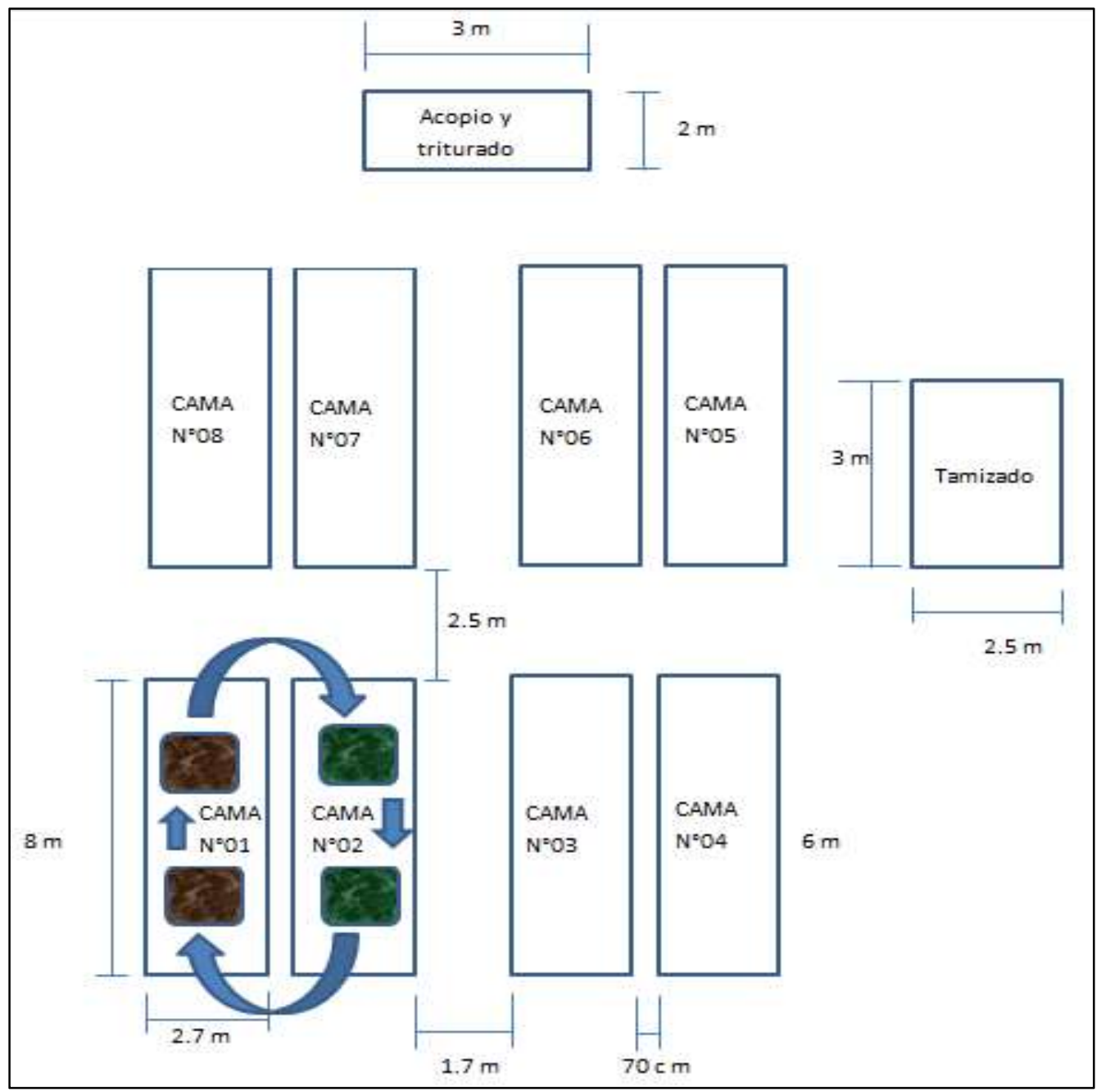

Figura 1: Nuevo sistema de la Planta de Compostaje. 


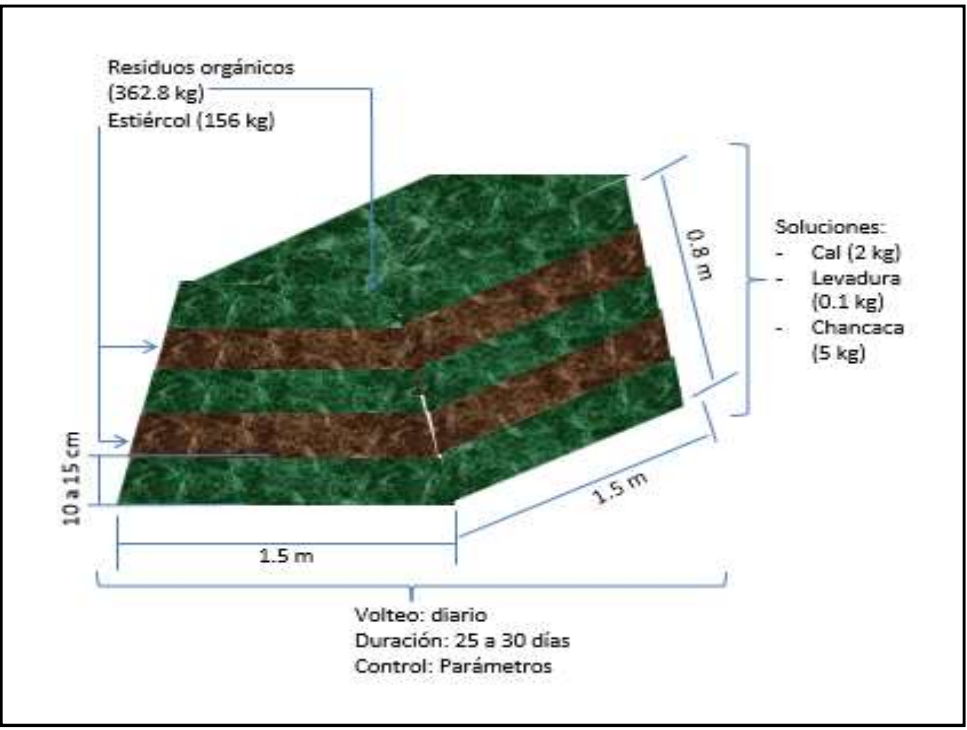

Figura 2: Diseño de pila de compostaje.

A. Diagrama de flujo del sistema:

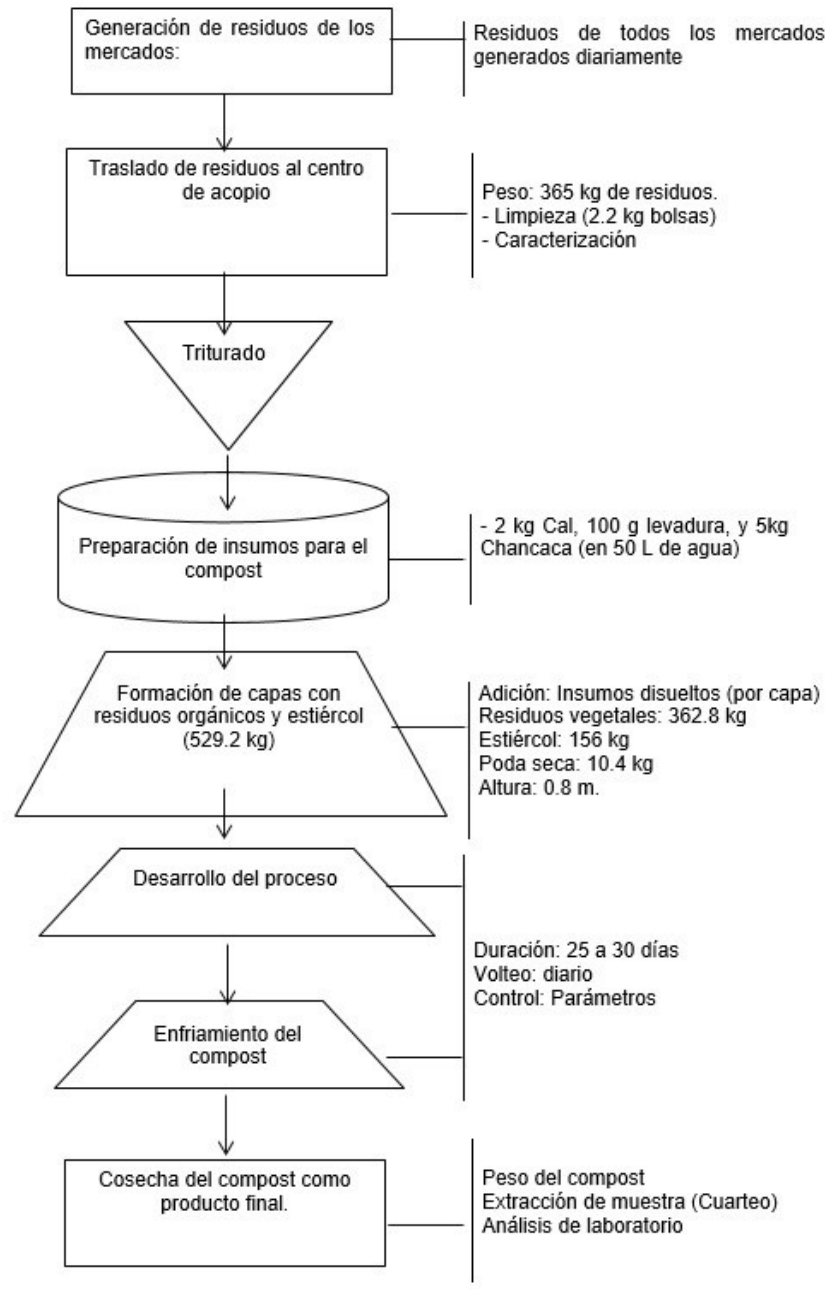

Figura 3: Diagrama de flujo. 
Tabla 1: Cantidad de residuos caracterizados.

\begin{tabular}{|c|c|c|c|}
\hline & & Peso $(\mathrm{kg})$ & $\%$ \\
\hline \multirow[t]{3}{*}{ Frutas } & Cítricos & 24,5 & 7 \\
\hline & Papaya & 16,9 & 5 \\
\hline & Otros & 31,4 & 8 \\
\hline \multirow[t]{3}{*}{ Verduras } & Hortalizas & 290 & 79 \\
\hline & Bolsas plásticas & 2,20 & 1 \\
\hline & Total & 365 & 100 \\
\hline
\end{tabular}

Se observa los pesos de acuerdo a las características de cada uno de ellos, siendo los de mayor presencia en toda la población de residuos orgánicos a compostar, Los resultados de estos pesos se analizaron en el programa Excel, como se muestra la figura 4.

En la Figura 4, se observa claramente que los residuos de mayor presencia son las verduras, denominadas hortalizas con $290 \mathrm{~kg}$, las cuales cada una de ellas se detalla en la Tabla 1. Entre las frutas se separó las que se observaban de mayor cantidad, las cuales se identificaron que en primer lugar fueron los cítricos, que pesaron $24,5 \mathrm{~kg}$; en segundo lugar la de mayor denominación se vio los residuos de papaya, con un valor de $16,9 \mathrm{~kg}$, y finalmente de acuerdo al grafico denominados otros residuos dieron un valor de $31,4 \mathrm{~kg}$, recalcar que en esta denominación se encuentran los residuos de frutas de poca cantidad de presencia, las cuales se encuentran las cascaras de tunas, plátanos, piña, restos de sandía, uva, durazno, manzana, entre otros. Cabe mencionar que estos provienen de juguerías y fruterías dentro del establecimiento. El peso total de residuos obtenidos es de $362,8 \mathrm{~kg}$ el cual fue la muestra para la elaboración de la pila. Así mismo mencionar también que se encontró 2,20 $\mathrm{kg}$ de bolsas plásticas, por lo que se tiene que retirar para que no cause ninguna alteración durante el proceso.

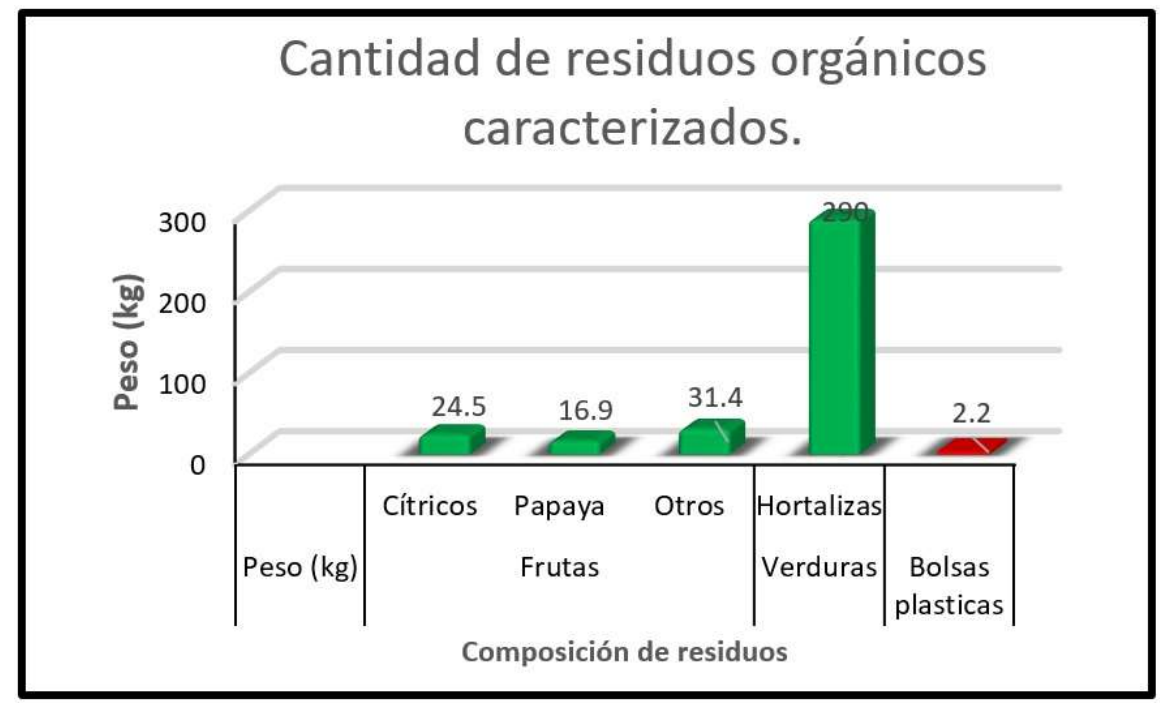

Figura 4: Cantidad de residuos orgánicos caracterizados.

2. Utilizar los insumos intervinientes para el sistema planteado en la producción de abono orgánico a partir de los residuos de frutas y verduras en la planta de tratamiento de la municipalidad de Comas.

En la figura 5 nos indica las cantidades exactas que se utilizaron para la elaboración del compost por el sistema planteado, el cual se tuvo como referencia el manual de abonos orgánicos de la FAO, 2013; las cuales fueron $156 \mathrm{~kg}$ de estiércol de vaca, 10,4 kg de poda, $2,5 \mathrm{~kg}$ de cal y $0,1 \mathrm{~kg}$ de levadura, las cuales facilitarán el desarrollo del proceso.

3. Medir las características fisicoquímicas en la producción de abono orgánico a partir de los 
residuos de frutas y verduras en la Planta de

Compostaje de la Municipalidad de Comas, 2018.

Tabla 2: Insumos para el compostaje.

\begin{tabular}{|l|r|}
\hline Insumos & Peso $(\mathrm{kg})$ \\
\hline Estiércol & 156 \\
\hline Poda seca & 10,4 \\
\hline Chancaca & 5,0 \\
\hline Cal & 2,5 \\
\hline Levadura & 0,1 \\
\hline
\end{tabular}

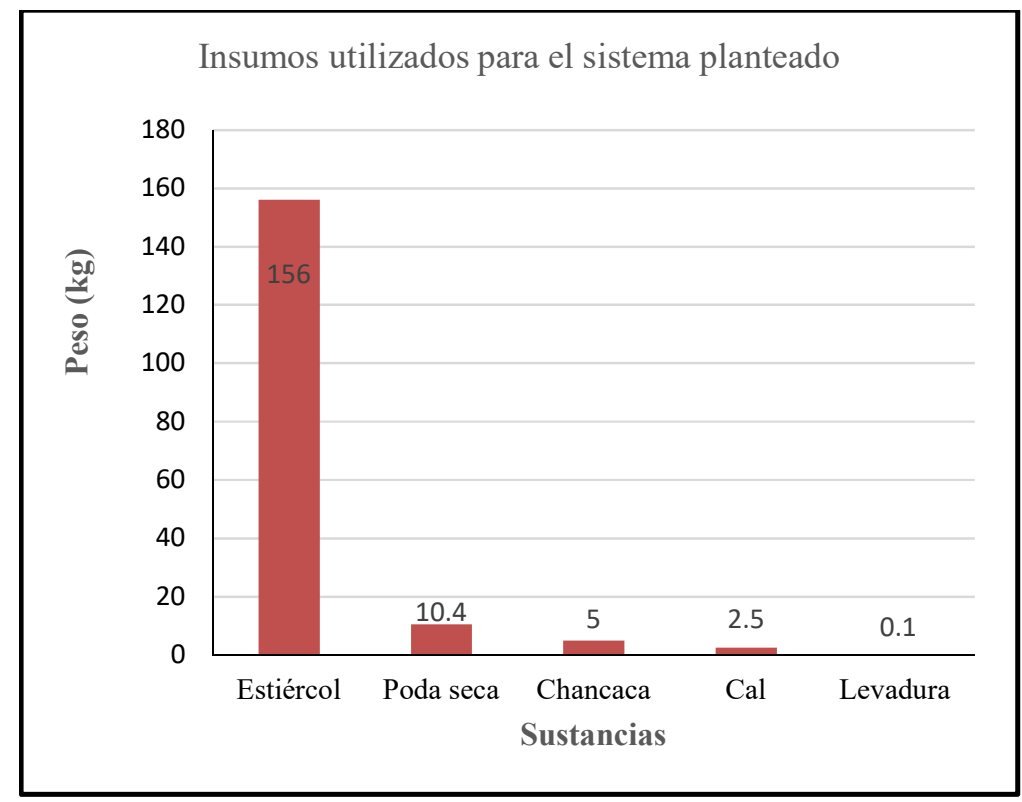

Figura 5: Cantidad de insumos utilizados para el sistema planteado.

Se observa registrado los tres parámetros de temperatura, $\mathrm{pH}$ y humedad con respecto a los volteos, estos valores se analizan en el programa Excel (Ver figura 6).

La figura 6 nos muestra claramente la variación de temperatura, esto se debe a que los dos primeros días fueron días de armado de pila, el cual el material orgánico que ingresa está fresca y por ende está a temperatura ambiente, conocida también como la fase mesófila; al cabo del tercer día el incremente es bastante notorio ya que como se observa en el gráfico la temperatura se eleva hasta los $58{ }^{\circ} \mathrm{C}$ dando lugar a la fase termófila, debido a que los microorganismos ya se activaron y empiezan la multiplicación entre ellos y la mayor degradación de la materia orgánica en esta fase. Con respecto a los demás parámetros no hay una un cambio significativo, por lo que el inicio de proceso está correcto.

Tabla 3: Valores obtenidos durante los primeros 4 días de la elaboración de la pila.

\begin{tabular}{|l|l|r|r|r|}
\hline \multicolumn{5}{|c|}{ SEMANA 0 } \\
\hline $\mathrm{N}^{\circ}$ Día & $\mathrm{N}^{\circ}$ Volteo & Temperatura $\left({ }^{\circ} \mathrm{C}\right)$ & $\mathrm{pH}$ & Humedad $(\%)$ \\
\hline 1 & 1 & 28 & 6,0 & 56,57 \\
\hline 2 & 1 & 28 & 6,0 & 56,39 \\
\hline 3 & 0 & 58 & 6,3 & 55,27 \\
\hline 4 & 0 & 60 & 6,5 & 53,98 \\
\hline
\end{tabular}


La Tabla $\mathrm{N}^{\circ}$ 04, muestra los valores desde el día cinco al onceavo, considerado semana 1.

En este periodo se empezó con los volteos frecuentes, los datos registrados se analizan en el programa Excel, ver figura 7.

La figura 7 se puede observar mediante la temperatura de esta semana la fase de desarrollo se mantiene en termófila ya que la temperatura se encuentra entre 54 a $65{ }^{\circ} \mathrm{C}$ siendo el valor más alto alcanzado el día seis, los demás días se observa una disminución mínima hasta el valor antes mencionado. Con el parámetro de $\mathrm{pH}$ se observa claramente en la Tabla 8 que en el día 10, hubo una disminución notoria, yéndose al ácido, por lo que para contrarrestar este déficit se le añadió $500 \mathrm{~g}$ de Cal para poder regularlo. Los demás parámetros no sufren ninguna alteración, marchando correctamente el desarrollo.

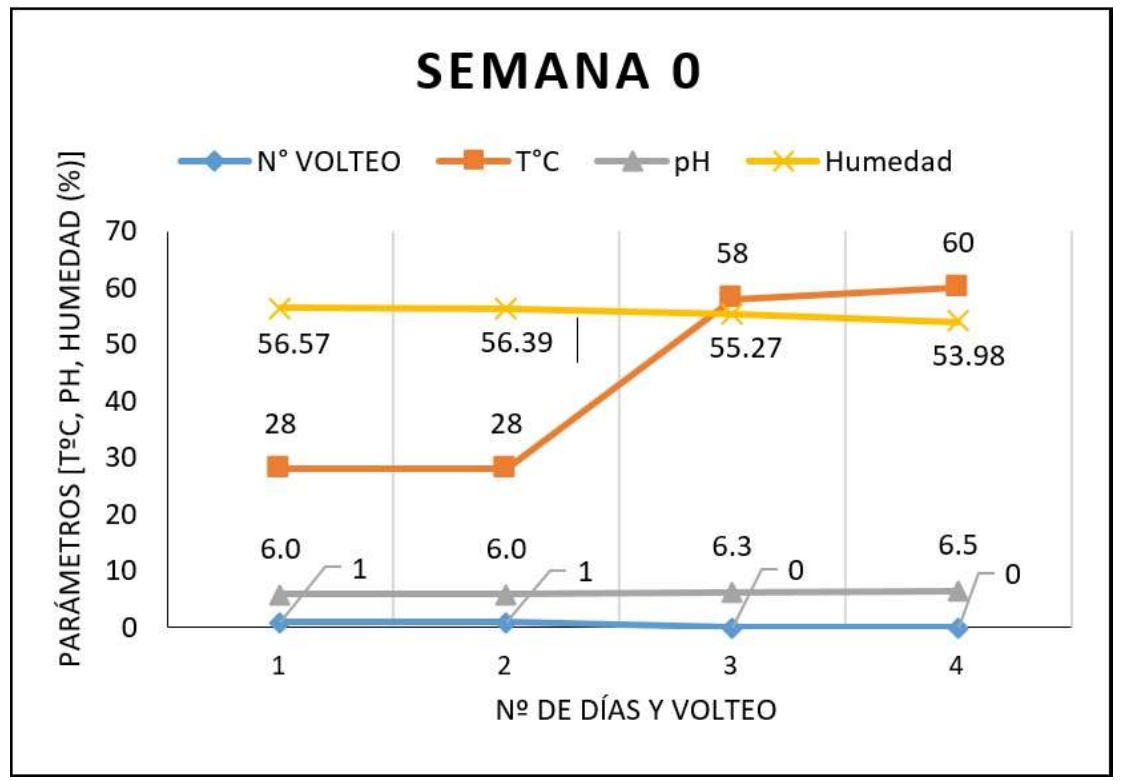

Figura 6: Monitoreo durante la semana 0.

La Tabla 5, muestra el registro de los datos del día 12 al 18 , considerada semana 02 .

La Figura 8 se observa que la temperatura va descendiendo conforme van pasando los días, desde los $52{ }^{\circ} \mathrm{C}$ hasta los $45^{\circ} \mathrm{C}$, en este parte del proceso se sigue considerando fase termófila ya que conservan aún temperaturas altas para el proceso, el volteo en este periodo es constante, el
$\mathrm{pH}$ se va acercando al neutro por lo que indica que está correcto, con respecto a la humedad va disminuyendo ligeramente conforme pasan los días.

La Tabla 6 muestra valores de la última semana de proceso, el cual se le consideró como semana 03, el cual se analiza y grafica en el programa Excel en la figura 9 .

Tabla 4: Monitoreo semana 1.

\begin{tabular}{|l|l|r|r|r|}
\hline \multicolumn{5}{|c|}{ SEMANA 1 } \\
\hline $\mathrm{N}^{\circ}$ Día & $\mathrm{N}^{\circ}$ Volteo & Temperatura $\left({ }^{\circ} \mathrm{C}\right)$ & $\mathrm{pH}$ & Humedad (\%) \\
\hline 5 & 1 & 62 & 6,5 & 53,11 \\
\hline 6 & 1 & 65 & 6,3 & 52,29 \\
\hline 7 & 1 & 64 & 6,3 & 50,97 \\
\hline 8 & 1 & 60 & 6,0 & 49,64 \\
\hline 9 & 1 & 57 & 6,0 & 48,53 \\
\hline 10 & 1 & 56 & 5,8 & 48,04 \\
\hline 11 & 1 & 54 & 6,2 & 47,85 \\
\hline
\end{tabular}


Tabla 5: Monitoreo semana 2.

\begin{tabular}{|l|l|r|r|r|}
\hline \multicolumn{5}{|c|}{ SEMANA 2 } \\
\hline $\mathrm{N}^{\circ}$ Día & $\mathrm{N}^{\circ}$ Volteo & ${\text { Temperatura }\left({ }^{\circ} \mathrm{C}\right)}$ & $\mathrm{pH}$ & Humedad (\%) \\
\hline 12 & 1 & 52 & 6,4 & 47,51 \\
\hline 13 & 1 & 48 & 6,4 & 47,23 \\
\hline 14 & 0 & 45 & 6,5 & 46,95 \\
\hline 15 & 0 & 46 & 6,5 & 46,57 \\
\hline 16 & 1 & 45 & 6,5 & 46,11 \\
\hline 17 & 0 & 45 & 6,7 & 45,98 \\
\hline 18 & 0 & 45 & 6,7 & 44,38 \\
\hline
\end{tabular}

SEMANA 1

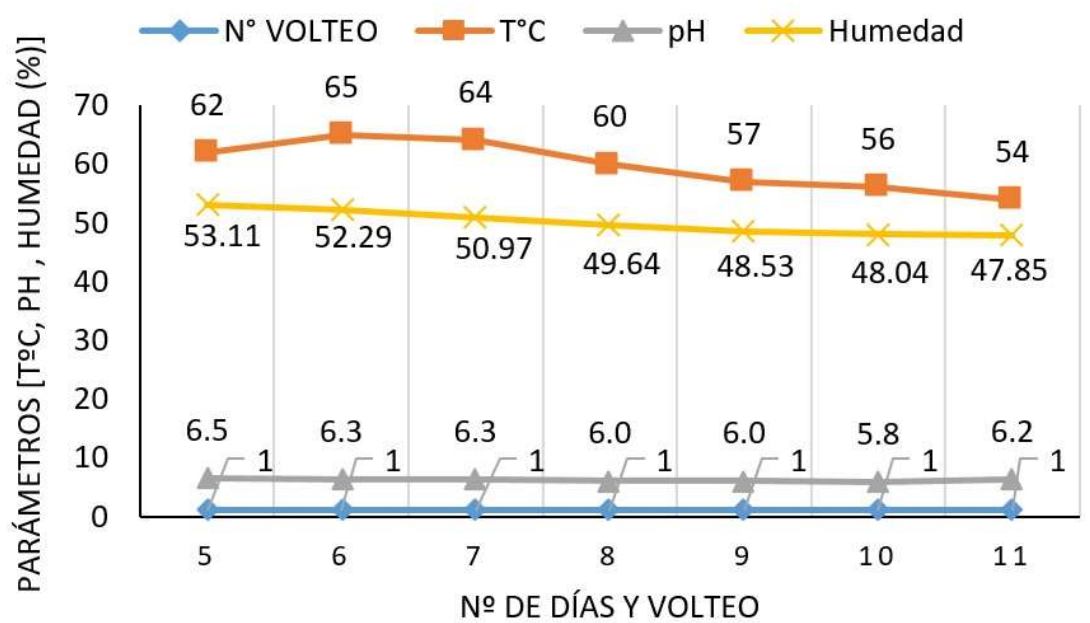

Figura 7: Monitoreo semana 1.

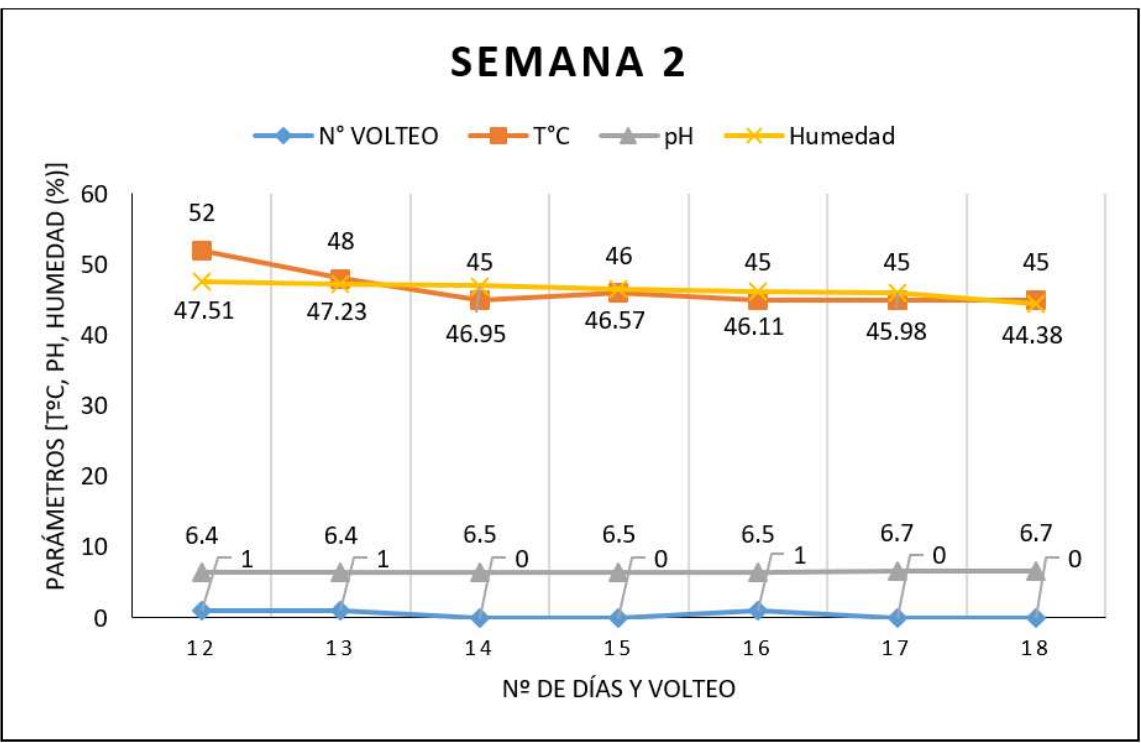

Figura 8: Monitoreo semana 2. 
Tabla 6: Monitoreo semana 3.

\begin{tabular}{|l|l|r|r|r|}
\hline \multicolumn{5}{|c|}{ SEMANA 3 } \\
\hline $\mathrm{N}^{\circ}$ Día & $\mathrm{N}^{\circ}$ Volteo & Temperatura $\left({ }^{\circ} \mathrm{C}\right)$ & $\mathrm{pH}$ & Humedad (\%) \\
\hline 19 & 0 & 43 & 6,7 & 43,06 \\
\hline 20 & 1 & 41 & 6,9 & 41,97 \\
\hline 21 & 0 & 35 & 6,9 & 39,90 \\
\hline 22 & 0 & 35 & 7,2 & 39,84 \\
\hline 23 & 1 & 36 & 7,2 & 39,75 \\
\hline 24 & 0 & 34 & 7,5 & 38,83 \\
\hline 25 & 0 & 32 & 7,5 & 38,02 \\
\hline 26 & 0 & 30 & 7,3 & 37,59 \\
\hline 27 & 0 & 29 & 7,3 & 36,55 \\
\hline
\end{tabular}

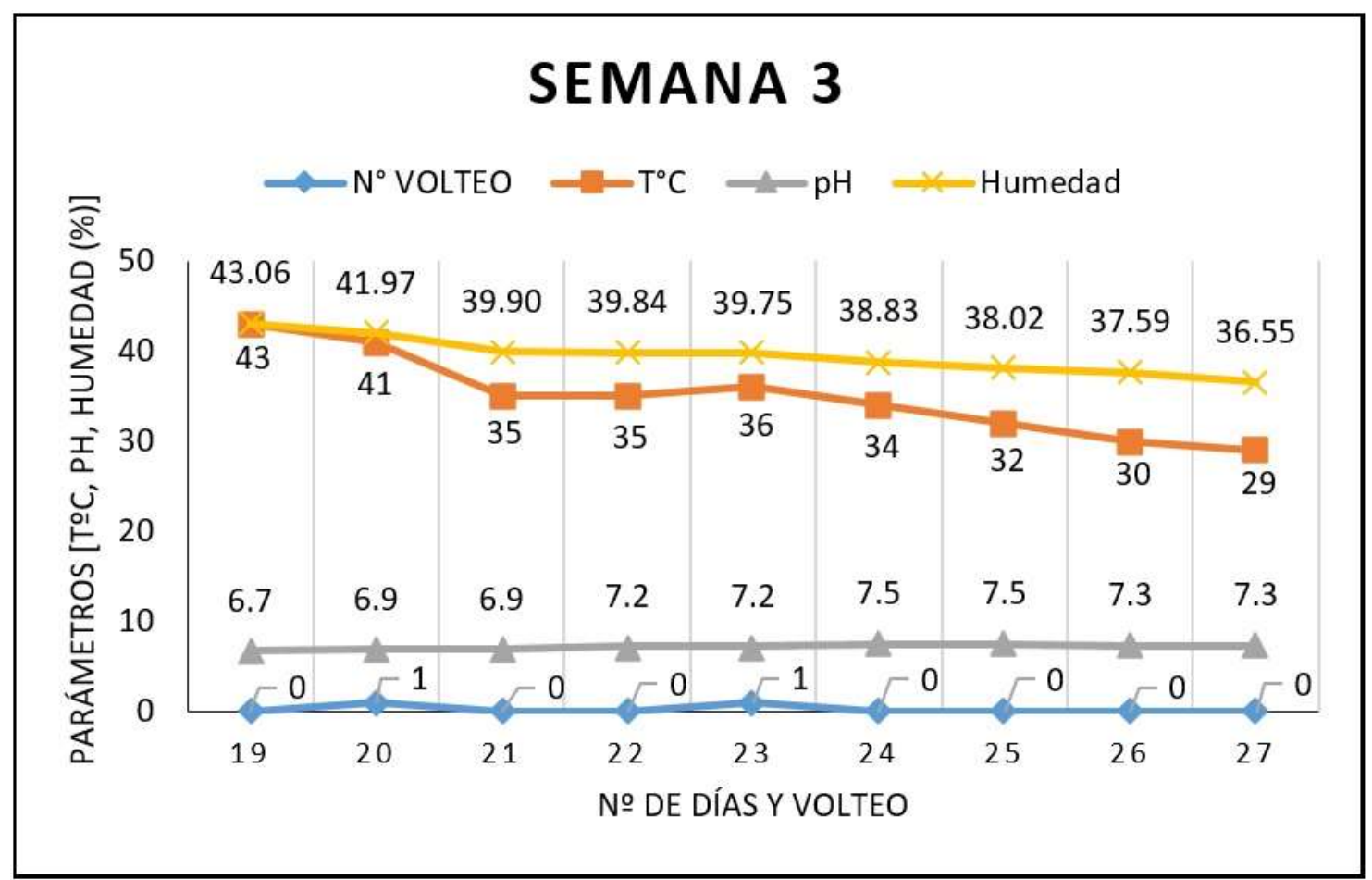

Figura 9: Monitoreo semana 03.

En la Figura 9, se ve claramente el descenso de la temperatura y que a partir del día 21 dejando la fase termófila para pasar a la fase de enfriamiento, ya que en esta etapa el compost empieza a estabilizarse tornando un color marrón oscuro y con olor a tierra de cultivo o chacra, el parámetro de humedad va disminuyendo ligeramente como se ve en la tabla 9, el pH se desarrolló conforme siempre cerca al neutro.

Finalmente, el día 27 que duró el proceso final ya que ese día se hizo el último monitoreo para después pasar el compost a la etapa del tamizado y seguidamente sacar la muestra del producto final para llevar ser analizado.

\subsection{Análisis estadístico de datos de monitoreo}

La presente investigación obtuvo datos durante el proceso de monitoreo de tal manera para analizar estos datos existen programas de tipo estadístico como el Excel, SPSS, en la que se ingresarán dichos datos de monitoreo para su posterior análisis.

Asimismo, se analizó en el programa SPSS los datos obtenidos durante la etapa experimental, para determinar el comportamiento de distribución normal, donde los valores de P-Valor son mayores a 0,05 , el cual indica que los datos están en un rango de $95 \%$ de confiabilidad. Para ello se realizó el análisis (Ver tabla 6). 
En la tabla 7 muestra el análisis los datos generados a través de toda la fase experimental, el cual obedecen a un comportamiento de una distribución normal, tomándose los valores de Shapiro-wilk debido a que son muestras pequeñas $(<30)$. Viendo los valores de $\mathrm{P}$-Valor son mayores a 0,05 , lo que está indicando que los datos están en el rango del $95 \%$ de confianza.

4. Evaluar la calidad del abono orgánico producido por el sistema planteado en la Planta de compostaje de la Municipalidad de Comas, 2018.
Los análisis de resultados de las muestras del compost se mandaron realizar en el laboratorio de suelos de la Universidad Nacional Agraria La Molina, del cual se detallará a continuación los análisis para evaluar la calidad del abono orgánico, analizando la presencia del $\mathrm{pH}$, Materia Orgánica y cada uno de los parámetros esenciales $(\mathrm{N}, \mathrm{P}, \mathrm{K})$.

La Figura 10, muestra el promedio de resultados del análisis de acuerdo a cada tratamiento realizado en la planta de compostaje de la municipalidad de Comas; para el parámetro del $\mathrm{pH}$.

Tabla 7: Prueba de normalidad de monitoreo de parámetros.

\begin{tabular}{|l|r|r|r|r|r|r|r|}
\hline \multirow{2}{*}{ Temperatura } & \multirow{2}{*}{ N $^{\circ}$ Volteos } & \multicolumn{4}{|c|}{ Kolmogorov-Smirnov } & \multicolumn{3}{|c|}{ Shapiro-Wilk } \\
\cline { 3 - 8 } & & Estadístico & gl & Sig. & Estadístico & gl & Sig. \\
\cline { 2 - 8 } & 0 &, 198 & 13 &, 172 &, 903 & 13 &, 147 \\
\hline \multirow{2}{*}{$\mathrm{pH}$} & 1 &, 144 & 14 &, $200^{*}$ &, 918 & 14 &, 207 \\
\cline { 2 - 8 } & 0 &, 216 & 13 &, 099 &, 900 & 13 &, 132 \\
\hline Humedad & 1 &, 177 & 14 &, $200^{*}$ &, 913 & 14 &, 171 \\
\cline { 2 - 8 } & 0 &, 192 & 13 &, $200^{*}$ &, 902 & 13 &, 141 \\
\hline
\end{tabular}

Tabla 8: Parámetro $\mathrm{pH}$.

\begin{tabular}{|c|c|r|}
\hline \multirow{3}{*}{$\mathrm{pH}$} & TMC_1 & 8,02 \\
\cline { 2 - 3 } & TMC_2 & 8,34 \\
\cline { 2 - 3 } & TMC_S & 7,04 \\
\hline
\end{tabular}

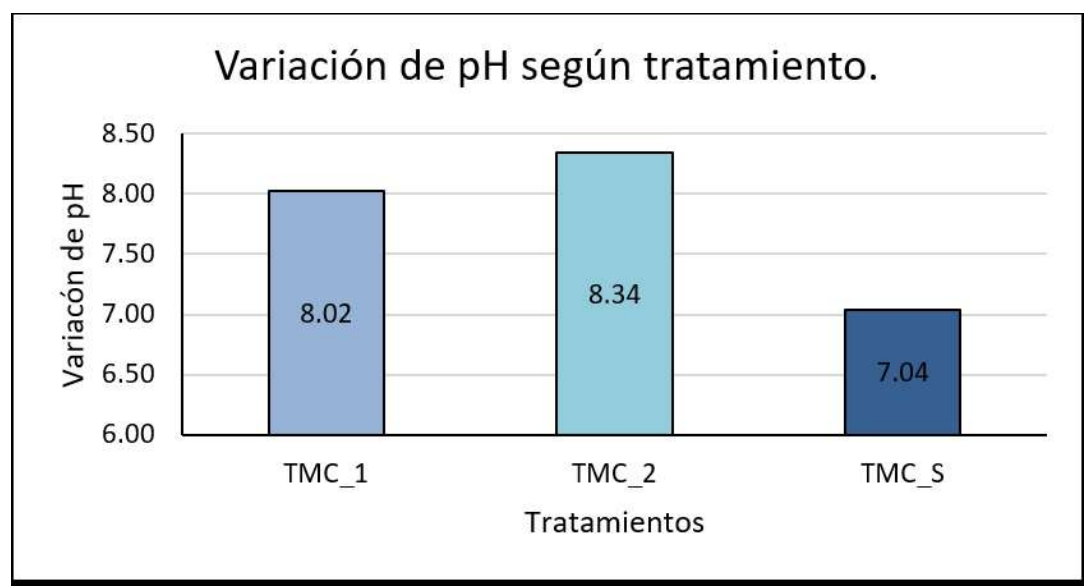

Figura 11: Promedio de variación de pH según tratamiento.

En la Figura 11, se observa la cantidad de los pH para cada muestra en los diferentes tratamientos, el cual el Tratamiento Municipalidad de Comas (TMC_1) y TMC_2 se encuentran por encima del valor 8,00 , considerándose según el manual de compostaje de la FAO dentro del rango permitido ya que indica que para este parámetro tiene que estar entre 6,5 a 8,5; pero considerando el tratamiento sistematizado (TMC_S) se ve un valor de 7,04, prácticamente estando bastante cerca al 7,00 , esto quiere decir que cuanto más se acerca al neutro tendrá mayor alcance hacia los cultivos, ya que la mayoría de ellas requiere un $\mathrm{pH}$ neutro para su desarrollo. 
La Figura 12, muestra promedio de los valores obtenidos en los resultados del compost para el parámetro Materia Orgánico (MO); las cuales se obtuvieron de la siguiente tabla.

En la Figura 12, se observa la cantidad de MO para cada muestra en los diferentes tratamientos, el cual el TMC 1 obtuvo un 31,06 \%; el TMC 2 alcanzó un valor de $31,96 \%$, con respecto al TMC_S indica que tiene un $32,32 \%$ de materia orgánica. Según el manual de compostaje de la FAO todos los resultados presentados se encuentran mayor a 20 \% (valor mínimo que requiere el compost) por lo que, al obtener mayor valor en el proceso sistematizado, mayor será la eficacia para mejorar y mantener el suelo.

La Figura 13, indica el promedio de los valores obtenidos en los resultados del compost para el parámetro de Nitrógeno $(\mathrm{N})$.

Tabla 9: Parámetro M.O. (\%).

\begin{tabular}{|l|r|r|}
\hline \multirow{2}{*}{ MO. } & TMC_1 & 31,06 \\
\cline { 2 - 3 } & TMC_2 & 31,96 \\
\cline { 2 - 3 } & TMC_S & 32,32 \\
\hline
\end{tabular}

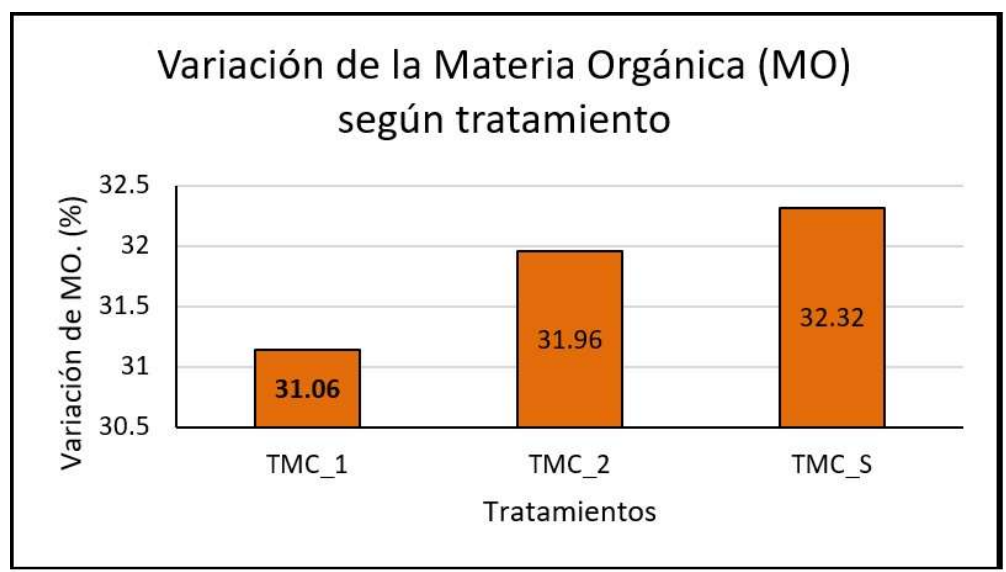

Figura 12: Promedio de variación de la Materia Orgánica (MO) según tratamiento.

Tabla 10: Parámetro N (\%).

\begin{tabular}{|c|r|r|}
\hline \multirow{3}{*}{$\mathrm{N}$} & TMC_1 & 1,40 \\
\cline { 2 - 3 } & TMC_2 & 1,52 \\
\cline { 2 - 3 } & TMC_S & 1,85 \\
\hline
\end{tabular}

En la Figura 13, se observa el nivel del Nitrógeno (N) para cada muestra en los diferentes tratamientos, el cual el TMC_1 obtuvo un 1,40 \%; el TMC_2 alcanzó un valor de $\overline{1}, 52 \%$, con respecto al TMC_S indica que tiene un 1,85\%. Según el manual de compostaje de la FAO todos los resultados presentados se encuentran dentro del rango permitido $(1 \%$ a $4 \%)$ el cual se puede observar que el tratamiento sistematizado (TMC_S) que se realizó obtuvo mayor valor el cual esto beneficiará más a la nutrición de los cultivos.

La Figura 14, menciona el promedio de los valores obtenidos de los resultados del compost para el parámetro Fósforo como (P2O5). 


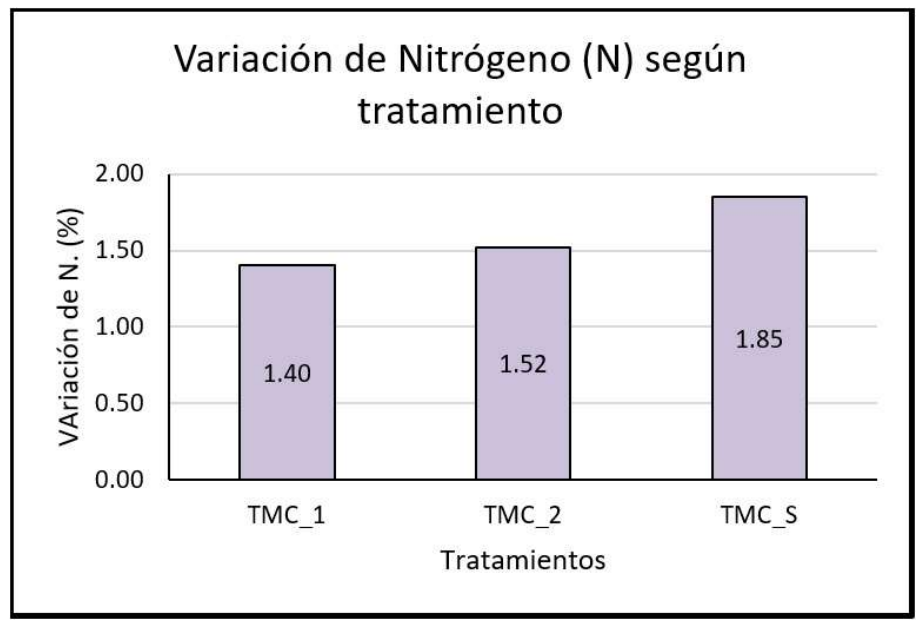

Figura 13: Promedio de variación de Nitrógeno $(N)$ según tratamiento.

Tabla 11: Parámetro P2O5 (\%).

\begin{tabular}{|l|r|r|}
\hline \multirow{3}{*}{$\mathrm{P}_{2} \mathrm{O}_{5}$} & TMC_1 & 1,54 \\
\cline { 2 - 3 } & TMC_2 & 1,72 \\
\cline { 2 - 3 } & TMC_S & 1,91 \\
\hline
\end{tabular}

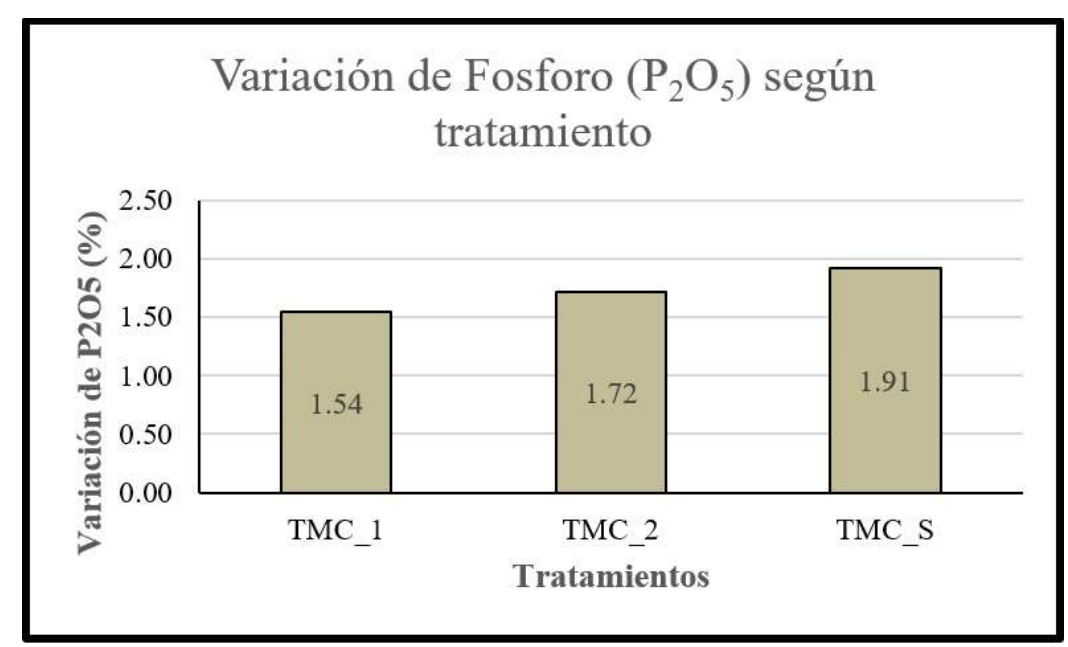

Figura 14: Promedio de variación del Fósforo (P2O5) según tratamiento.

En la Figura 14, se observa el nivel del Fósforo $\left(\mathrm{P}_{2} \mathrm{O}_{5}\right)$ para cada muestra en los diferentes tratamientos, el cual el TMC_1 obtuvo un 1,54 \%; el TMC_2 alcanzó un valor de $\overline{1}, 72 \%$, con respecto al TMC_S indica que tiene un 1,91\%. El cual se puede observar que el tratamiento sistematizado (TMC_S) que se realizó obtuvo mayor valor con respecto a los demás, el cual tendrá buena aportación para el desarrollo y eficacia de la fotosíntesis de las plantas.

La Figura 15 indica el promedio de los valores obtenidos de los resultados del compost para el parámetro Potasio como $\left(\mathrm{K}_{2} \mathrm{O}\right)$.

Tabla 12: Parámetro $\mathrm{K}_{2} \mathrm{O}(\%)$.

\begin{tabular}{|l|r|r|}
\hline \multirow{2}{*}{$\mathrm{K}_{2} \mathrm{O}$} & TMC_1 & 1,46 \\
\cline { 2 - 3 } & TMC_2 & 1,57 \\
\cline { 2 - 3 } & TMC_S & 3,19 \\
\hline
\end{tabular}




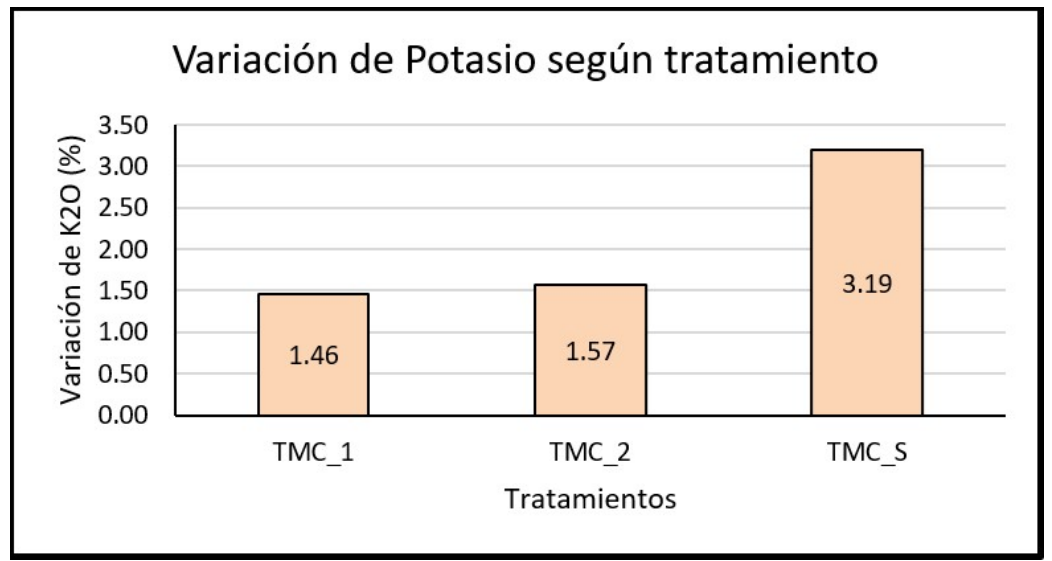

Figura 15: Promedio de variación del Potasio (K2O) según tratamiento.

En la Figura 15, se observa el nivel del Potasio $\left(\mathrm{K}_{2} \mathrm{O}\right)$ para cada muestra en los diferentes tratamientos, el cual el TMC_1 obtuvo un 1,46\%; el TMC_2 alcanzó un valor de 1,57\%, con respecto al TMC_S indica que tiene un 3,19\%. El cual se puede observar que el tratamiento sistematizado (TMC_S) que se realizó obtuvo mayor valor con respecto a los demás, el cual tendrá buena aportación para el desarrollo y eficacia de la fotosíntesis de las plantas.

\subsection{Análisis general de parámetros}

En la Tabla 13, indica todos los resultados por cada parámetro obtenidos del análisis en laboratorio del compost de la municipalidad de Comas.

En la Figura 16 los valores obtenidos representado gráficamente de acuerdo a cada parámetro según los tratamientos de cada uno, se observa que el Tratamiento de la Municipalidad de Comas Sistematizado (TMC_S) obtuvo una ventaja con respecto a los demás tratamientos, por lo que este proceso sería el más adecuado para la producción del abono orgánico, ya que el tiempo de desarrollo fue más rápido, ejecutándose en 27 días y logrando conservar y mejorar la calidad del compost que se produce.

\section{Discusión y conclusiones}

\subsection{Discusión}

En la investigación durante la etapa de caracterización de los tipos de residuos consistió en separar las frutas y verduras, identificando cada una de ellas mediante la observación, para luego tomar el peso de aquellos tipos que presentan mayor volumen; asimismo segregar los materiales inorgánicos como bolsas. Contrastando con el artículo de [1] titulada Perspectivas de aplicación del compostaje de bioresiduos provenientes de residuos municipales, un enfoque desde lo global a lo local; menciona estrategias de separación en la fuente de recolección selectiva para después posicionar los residuos de manera adecuada para su posterior tratamiento; se deduce que, al caracterizar los residuos, estas se segregan para evitar algunas alteraciones durante el proceso de desarrollo del compostaje.

Tabla 13: Datos generales de los parámetros.

\begin{tabular}{|l|r|r|r|r|r|}
\hline & \multicolumn{3}{|c|}{ Parámetros (\%) } \\
\hline Tratamientos & $\mathrm{pH}$ & $\mathrm{MO}$. & $\mathrm{N}$ & $\mathrm{P} 2 \mathrm{O} 5$ & $\mathrm{~K} 2 \mathrm{O}$ \\
\hline TMC_1 & 8,2 & 31,14 & 1,4 & 1,55 & 1,46 \\
\hline TMC_2 & 8,34 & 31,96 & 1,52 & 1,72 & 1,57 \\
\hline TMC_S & 7,04 & 32,32 & 1,92 & 1,92 & 3,19 \\
\hline
\end{tabular}




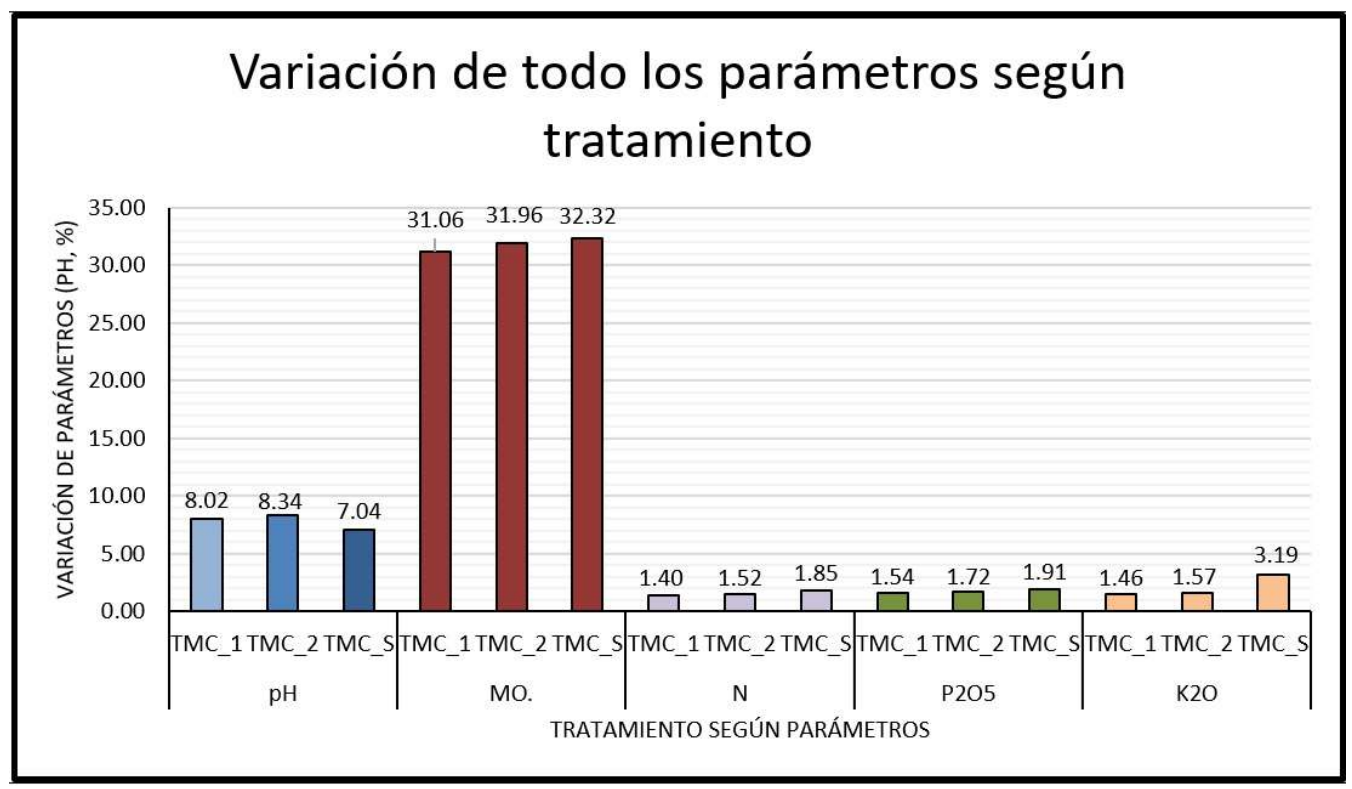

Figura 16: Variación general de los parámetros según tratamiento.

Los insumos intervinientes, como la cal, levadura, estiércol son de gran ayuda ya que de ellos se depende la neutralización del $\mathrm{pH}$, población microbiana para degradación de la materia orgánica y aportes de suplementos que aportan los nutrientes del compost. En comparación con el autor [2] en su artículo "Influence of Locally Additives on Neem Plant Organic Fertilizer Quality in Samaru,) Zaria, Kaduna State". (Influencia de los aditivos locales en la calidad del fertilizante orgánico de plantas de Neem en Samaru, Zaria, estado de Kaduna, Nigeria) tuvo como objetivo evaluar la influencia de los aditivos de origen local en la calidad del fertilizante orgánico; se introdujeron aditivos en la producción para mejorar la calidad del fertilizante, como la piedra caliza, para neutralizar el suelo, litros de aves de corral como suplemento del contenido de nitrógeno, fosforo y potasio, fosfato de roca para estandarizar el contenido de fósforo del suelo y retener la textura y composición del suelo. Se deduce que los insumos utilizados son de gran importancia para el proceso de desarrollo ya que aportan suplementos como el nitrógeno que encontramos en el estiércol, el fosforo y potasio en la chancaca, y cal para el uso de neutralización del $\mathrm{pH}$ y poder llevar el control adecuado del desarrollo del compostaje.

Con respecto al control de las condiciones fisicoquímicas se tomó en cuenta los parámetros principales para el desarrollo del proceso $(\mathrm{pH}$, temperatura y humedad) siendo los tres factores muy importantes; la frecuencia de volteos y monitoreo de parámetros fueron diario, mejorando el tiempo de degradación de la materia orgánica y logrando resultados en 27 días desde su inicio de proceso. En comparación con Álvarez, [et al] (2013) en su artículo titulada Rediseño y optimización de un dispositivo de compostaje a pequeña escala para ser utilizado en proyectos de agricultura urbana, donde menciona después de rediseñar el dispositivo de compostaje teniendo en cuenta siempre los factores que controlan el proceso aeróbico del compostaje (oxígeno, $\mathrm{pH}$, humedad y temperatura), se procedió a la toma diaria de los parámetros de temperatura, $\mathrm{pH}$ y humedad, como punto de partida para el inicio del proceso de compostaje que tuvo una duración de 28 días a partir del día de actividades de iniciación del proceso; se deduce que controlando los parámetros del $\mathrm{pH}$, humedad y temperatura son factores muy determinantes para mejorar el tiempo en la obtención del compost.

De acuerdo a la evaluación de la temperatura durante el desarrollo del proceso alcanzó valores altos hasta $65^{\circ} \mathrm{C}$ esto se debe a que tuvo una altura adecuada de tamaño de pila con $80 \mathrm{~cm}$ de altitud, el cual tuvo un desarrollo adecuado y acorde a los rangos descritos por la FAO, contrastando con [4], en su tesis titulada Optimización del proceso de compostaje de pequeña escala el cual tuvo como objetivo optimizar un sistema de compostaje de pequeña escala para que tenga la capacidad de adaptarse a regiones con climas fríos y permita tener una mejora en la calidad agronómica, para ello su tratamiento con altura de 60 a $80 \mathrm{~cm}$ de altura tuvo mejores resultados durante el desarrollo del 
proceso; se puede constatar que la altura que se manejó en esta investigación también fue de $80 \mathrm{~cm}$ por lo que resulto bien durante todo el proceso, alcanzando temperaturas adecuadas y eficientes para la degradación de este.

Con respecto a la evaluación de la calidad del compostaje obtenido por el sistema planteado se comprobó resultados de las tres muestras analizadas, las cuales fueron obtenidos de los diferentes tratamientos, para el TMC_1 (Tratamiento de la Municipalidad de Comas) se obtuvo $8,20 \mathrm{pH}, 31,06$ M.O., 1,40 N, 1,54 P y 1,46 K. Para el TMC_2 fueron 8,34 pH, 31,96 M.O., 1,52 $\mathrm{N}, 1,72 \mathrm{P}$ y $1,57 \mathrm{~K}$. $\mathrm{Y}$ finalmente con el sistema planteado TMC_S se consiguió 7,04 pH, 32,32 M.O., $1,85 \mathrm{~N}, 1, \overline{9} 1 \mathrm{P}$ y $3,19 \mathrm{~K}$ lográndose observar una diferencia entre ellas, el cual el tratamiento planteado mostró mejoras en los resultados. Contrastando con [5] el cual utilizó una técnica de digestión de la materia orgánica el cual resalta la obtención del compost con residuos vegetales y microorganismos eficientes. Consiguiendo compost con $\mathrm{N}(0,96 \%)$, fosforo $(0,86 \%)$, Potasio $(1,63 \%)$. En contenido de materia orgánica, se registró un porcentaje final de $27,48 \%$. que ayuda bastante en sus aportes a los suelos. Así mismo se deduce que los valores obtenidos con el proceso sistematizado se ganaron ligeramente en la cantidad de nutrientes el cual beneficiará mejor al suelo.

\subsection{Conclusiones}

1. La identificación de los tipos de residuos orgánicos presentes en las frutas y verduras en la Planta de compostaje de la municipalidad de Comas, 2018; mayormente ingresan residuos vegetales, el cual tuvo un peso de $290 \mathrm{Kg}$, representando el $79 \%$, con respecto a las frutas el $7 \%$ fueron los cítricos, $5 \%$ residuos de papaya, $8 \%$ considerado otros a los residuos de frutas de presencias unitarias y $1 \%$ de residuos de bolsas plásticas.

2. Con respecto a la inoculación de insumos se utilizó e1 $56 \mathrm{~kg}$ de estiércol el cual es un aporte rico en nitrógeno, $5 \mathrm{~kg}$ de Chancaca ayuda como suplemento de fosforo y potasio, $100 \mathrm{~g}$. de Levadura como aporte en la población microbiana para degradación de la materia orgánica, $10,4 \mathrm{~kg}$ de poda seca para el uso como base de la pila de compost y poder cubrir toda la pila amada y finalmente los 2,5 $\mathrm{kg}$ de Cal para controlar el pH durante el proceso.
3. Las condiciones de operación en las características fisicoquímicas, la fase termófila tuvo una duración de 16 días alcanzando una temperatura máxima de $64^{\circ} \mathrm{C}$ y mínima de $45^{\circ} \mathrm{C}$. La fase de enfriamiento o también conocido como mesófila II se desarrolló en un periodo de 9 días el cual un indicador de esta fase es el descenso de temperatura desde $43{ }^{\circ} \mathrm{C}$ hasta $29{ }^{\circ} \mathrm{C}$, donde se concluye que el tiempo de producción tuvo lugar en 27 días logrando convertir la materia prima de frutas y verduras en abono orgánico.

4. Los resultados obtenidos mejoraron con el sistema planteado, con respecto al $\mathrm{pH}$ se obtuvo un valor 7,04 el cual es más cercano al neutro; la Materia Orgánica (MO.) se llegó obtener 32,32 \% mejorando $1 \%$ más respecto a los otros; El Nitrógeno $(\mathrm{N})$ llegó a 1,92 \% también con una diferencia de $1 \%$ más respecto a los demás en este tratamiento; el Fosforo (P2O5) 1,92 \% siendo mejor que los otros dos resultados y finalmente el Potasio (K2O) con 3,19\% incrementando $2 \%$ respecto a los otros dos resultados de los tratamientos normales que se realizan. El cual se obtuvo un abono de mejor calidad ya que se consiguió mejorar cada uno de los parámetros que son esenciales para la asimilación de los cultivos.

\section{Referencias}

[1] R. Oviedo, L. Marmolejo y P. Torres, Revista de Ingeniería 1 (2012) 67-75.

[2] D. Ibrahim, A.A. Danmalam, A.I. Salihu y U.M. Jajere, Journal of Applied Sciences and Environmental Management 22 (2018) 8.

[3] J. Álvarez, C. Gómez, F. Herrera y M.C. Echavarria, Revista Elementos 3 (2013).

[4] J. Arrigoni, Tesis, Universidad Nacional de Córdova, 2016.

[5] E. Larreategui y C. Banchón, Enfoque UTE, 5 (2014) 2.

\section{E-mail:}

omargv10@hotmail.com, eacostas@ucv.edu.pe aliagamartinez@yahoo.com, ebenitesa@ucv.edu.pe 